ARTICLE

https://doi.org/10.1038/s41467-019-09686-v/

\title{
Geometric imaging of borophene polymorphs with functionalized probes
}

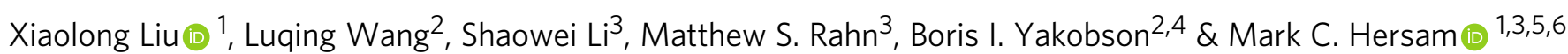

A common characteristic of borophene polymorphs is the presence of hollow hexagons $(\mathrm{HHs})$ in an otherwise triangular lattice. The vast number of possible $\mathrm{HH}$ arrangements underlies the polymorphic nature of borophene, and necessitates direct $\mathrm{HH}$ imaging to definitively identify its atomic structure. While borophene has been imaged with scanning tunneling microscopy using conventional metal probes, the convolution of topographic and electronic features hinders unambiguous identification of the atomic lattice. Here, we overcome these limitations by employing $\mathrm{CO}$-functionalized atomic force microscopy to visualize structures corresponding to boron-boron covalent bonds. Additionally, we show that COfunctionalized scanning tunneling microscopy is an equivalent and more accessible technique for $\mathrm{HH}$ imaging, confirming the $v_{1 / 5}$ and $v_{1 / 6}$ borophene models as unifying structures for all observed phases. Using this methodology, a borophene phase diagram is assembled, including a transition from rotationally commensurate to incommensurate phases at high growth temperatures, thus corroborating the chemically discrete nature of borophene.

\footnotetext{
${ }^{1}$ Applied Physics Graduate Program, Northwestern University, Evanston, IL 60208, USA. ${ }^{2}$ Department of Materials Science and NanoEngineering, Rice University, Houston, TX 77005, USA. ${ }^{3}$ Department of Materials Science and Engineering, Northwestern University, Evanston, IL 60208, USA. ${ }^{4}$ Department of Chemistry, Rice University, Houston, TX 77005, USA. ${ }^{5}$ Department of Chemistry, Northwestern University, Evanston, IL 60208, USA. ${ }^{6}$ Department of Electrical Engineering and Computer Science, Northwestern University, Evanston, IL 60208, USA. Correspondence and requests for materials should be addressed to M.C.H. (email: m-hersam@northwestern.edu)
} 
T he recent experimental realization of $2 \mathrm{D}$ boron ${ }^{1-8}$ (i.e., borophene) has spurred broad interest in its unique material attributes such as in-plane anisotropy ${ }^{9}$, massless Dirac fermions ${ }^{10}$, and seamless phase intermixing ${ }^{7}$. The polymorphic nature of borophene 2,3 , which is rooted in the rich bonding configurations among boron atoms, further distinguishes it from other $2 \mathrm{D}$ materials and offers an additional means for tailoring its material properties. However, when combined with the large number of predicted lattice structures for borophene, polymorphism introduces challenges for the unambiguous identification of borophene atomic structures experimentally. For example, while conventional metal-tip scanning tunneling microscopy (STM) has provided atomic-scale characterization of borophene $e^{4-7}$, the convolution of topographic and electronic structure in the resulting images coupled with Moiré superlattices with the underlying substrate leads to experimental uncertainties that do not provide sufficient differentiation among possible borophene lattices. While comparison of available experimental data with density functional theory calculations and simulated images help exclude some possibilities, sufficient ambiguity remains such that the literature lacks consensus for many observed borophene phases ${ }^{5,11}$. Consequently, an experimental method for definitively identifying borophene atomic structures is needed in order to establish the fundamental structure-property relationships that underlie emerging borophene applications. Since the first evidence of enhanced scanning probe spatial resolution with pentacene-functionalized tips ${ }^{12}$, deliberate tip functionalization with $\mathrm{CO}^{13}, \mathrm{H}_{2}{ }^{14,15}, \mathrm{Xe}^{16}$, and $\mathrm{CuO}_{\mathrm{x}}{ }^{17,18}$ has been utilized to resolve spatial details down to the subatomic regime. Although the physical mechanisms that lead to this improved spatial resolution is still under debate ${ }^{19}$, geometric imaging with functionalized tips reveals inter-molecular and intra-molecular chemical bonds, particularly in carbon-based molecular or graphitic systems ${ }^{20-22}$.

In an extension of this methodology, we show here that cryogenic ultrahigh vacuum non-contact $\mathrm{CO}$-functionalized atomic force microscopy (CO-AFM) geometrically resolves features that are consistent with boron-boron covalent bonds. We further demonstrate that CO-functionalized STM (CO-STM $)^{22}$ resolves borophene $\mathrm{HHs}$ and thus provides equivalently unambiguous geometric identification of borophene atomic lattice structures in a manner that is experimentally less demanding. By applying this approach to several borophene phases produced at different growth temperatures, a borophene phase diagram is constructed that includes multiple borophene polymorphs with different rotational alignments on $\mathrm{Ag}(111)$. In particular, a transition from rotationally commensurate to rotationally incommensurate borophene phases is observed at high growth temperatures, which suggests that borophene is a chemically discrete two-dimensional material as opposed to a surface reconstruction or alloy with the underlying $\mathrm{Ag}(111)$ growth substrate.

\section{Results}

Non-rotated borophene phases. Borophene polymorphs are characterized by different arrangements and concentrations of $\mathrm{HHs}$ in an otherwise 2D triangular lattice. Figure 1a shows a conventional bare-tip STM image of intermixed $v_{1 / 5}$ (colored blue) and $v_{1 / 6}$ (colored red) phase borophene grown at a previously established temperature of $\sim 450^{\circ} \mathrm{C}^{7}$. By comparing these borophene structures with the atomic lattice of the $\mathrm{Ag}(111)$ growth substrate (Fig. 1b), the horizontal HH row directions of both phases are found to be $30^{\circ}$ rotated with respect to the $\mathrm{Ag}$ atomic chains (directions $\boldsymbol{a}$ or $\boldsymbol{b}$ in Fig. 1c), consistent with previously proposed structural models ${ }^{4}$ as shown in Fig. 1c. For $v_{1 / 5}$ and $v_{1 / 6}$ phase borophene, the HHs are arranged in a staggered and aligned manner, respectively, with the unit cells of the HH lattices indicated by the black arrows. High-resolution STM imaging of $v_{1 / 5}$ phase borophene with a bare metal tip (Fig. 1d) reveals a strong brick-wall type Moiré pattern modulating finer features.

In an effort to gain higher spatial resolution, imaging of these phases was repeated with CO-AFM. In particular, Figure 1e shows the characteristic inelastic electron tunneling spectra of $\mathrm{CO}$ obtained on borophene after decorating the metal tips with a $\mathrm{CO}$ molecule (hindered translation and rotation modes at $3.2 \mathrm{meV}$ and $17.6 \mathrm{meV}$, respectively), confirming successful tip functionalization (Supplementary Fig. 1) ${ }^{23}$. In contrast to the STM image in Fig. 1d, the constant height CO-AFM image in Fig. If geometrically resolves the $\mathrm{HH}$ lattice and verifies the proposed $v_{1 / 5}$ phase borophene structure, where the HHs are separated by bright ridges at positions expected for boron-boron covalent bonds. In agreement with the experimental image, Figure $1 \mathrm{~g}$ shows a simulated CO-AFM image ${ }^{24,25}$ with an overlaid $v_{1 / 5}$ phase atomic structure. While the apparent bonding structures of the 6-membered boron rings (i.e., HHs) are clearly resolved, minimal contrast is observed for the triangular boron lattice, where only 3 -membered boron rings exist. This limited spatial resolution is reasonable given the fact that 4 -membered carbon rings have also not been resolved with $\mathrm{CO}-\mathrm{AFM}^{26}$. Nevertheless, resolving the $\mathrm{HH}$ lattice is sufficient for determining the structures of borophene polymorphs and thus CO-AFM is a definitive tool for identifying borophene atomic lattice structures $^{3}$.

In light of the highly demanding nature of non-contact AFM in terms of instrumentation and operation, we further demonstrate that more experimentally accessible CO-STM imaging provides equivalent geometric information in Fig. 1h. Compared to the bare-tip STM image in Fig. 1d, a lattice of staggered protrusions (red arrows) matching the $\mathrm{HH}$ lattice are clearly resolved in addition to the less-obvious Moiré pattern. By performing COSTM imaging in dynamic mode ${ }^{27}$ (i.e., with an oscillating STM tip), this contrast is further enhanced (Fig. 1i) at milder imaging conditions (Supplementary Fig. 2), which helps improve the stability of the adsorbed $\mathrm{CO}$ molecule on the tip apex.

$30^{\circ}$-rotated borophene phases. At higher growth temperatures $\left(\sim 500^{\circ} \mathrm{C}\right), v_{1 / 5}$ phase borophene dominates ${ }^{7}$, although another phase with a rectangular lattice also begins to appear (Fig. 2a). This structure was first observed in high-temperature growth and assigned to a buckled triangular lattice ${ }^{5}$. The $3 \AA \times 5 \AA$ lattice constants are also indicative of $v_{1 / 6}$ phase borophene, which normally grows at lower temperatures ${ }^{4}$. However, the absence of a linear Moiré pattern typically seen for $v_{1 / 6}$ phase borophene suggests that this phase might be the $v_{1 / 6}$ sheet on $\operatorname{Ag}(111)$ with a different orientation with respect to the underlying $\operatorname{Ag}(111)$ lattice as suggested previously ${ }^{28,29}$. Indeed, instead of being parallel as in the low-temperature $v_{1 / 6}$ phase (Fig. 1c), the $\mathrm{HH}$ rows in this case are perpendicular to those of a neighboring $v_{1 / 5}$ borophene domain in Fig. $2 \mathrm{~b}$ (yellow arrows). We thus label this phase as $v_{1 / 6}$ $-30^{\circ}$ as schematically shown in Fig. $2 \mathrm{c}$, indicating that the borophene lattice orientation is rotated by $30^{\circ}$ on $\operatorname{Ag}(111)$ compared to the low-temperature $v_{1 / 6}$ phase.

A polycrystalline domain of the $v_{1 / 6}-30^{\circ}$ phase with $60^{\circ}$ grain boundaries (GBs; yellow arrows) is shown in Fig. 2d. Figure 2e, $\mathrm{f}$ are high-resolution STM and CO-AFM images of $v_{1 / 6}-30^{\circ}$ phase borophene, respectively, where the aligned $\mathrm{HHs}$ are clearly resolved in Fig. 2f with neighboring HHs separated by bright ridges at positions expected for boron-boron covalent bonds. In Fig. $2 \mathrm{~g}$, the simulated CO-AFM image with an overlaid atomic 
a

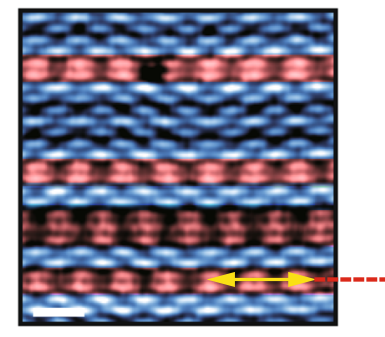

d

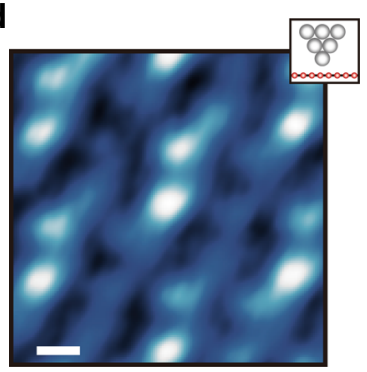

g

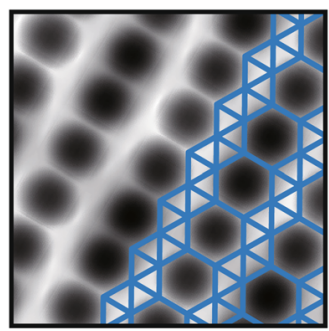

b

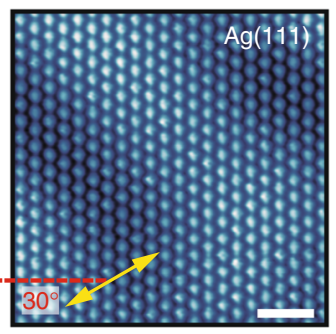

e

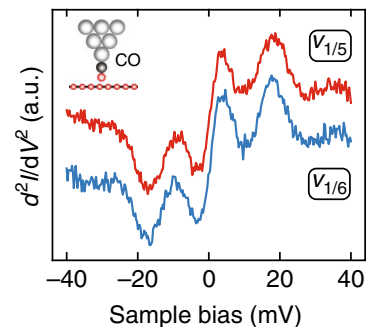

h

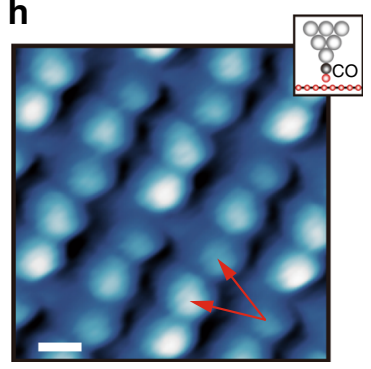

C

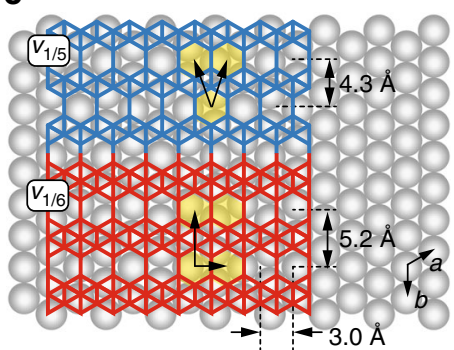

f
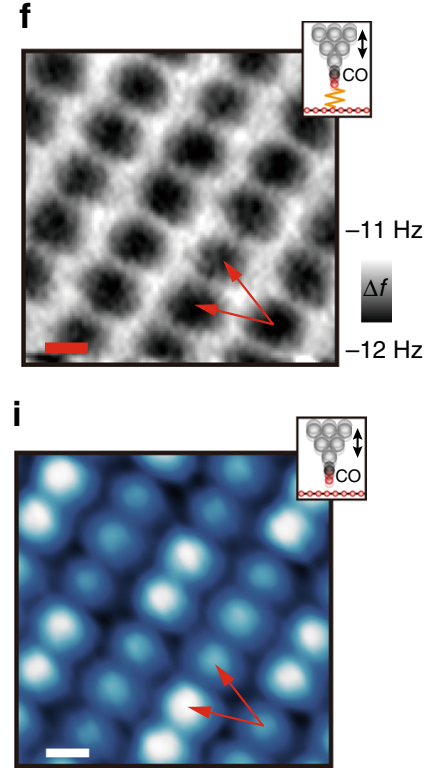

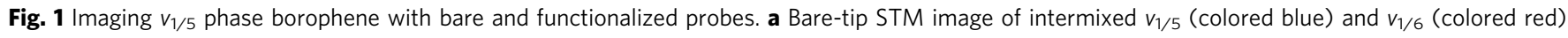
phase borophene. $\mathbf{b} \mathrm{A}$ neighboring $\mathrm{Ag}(111)$ surface, where the angle between the $\mathrm{HH}$ rows of borophene (horizontal) in a and the $\mathrm{Ag}$ atomic chains is $30^{\circ}$

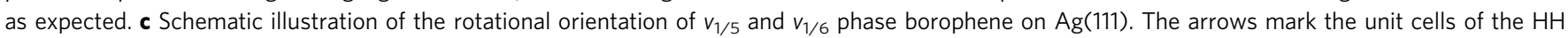
lattices in each phase. d Bare-tip STM image of $v_{1 / 5}$ phase borophene. e Inelastic electron tunneling spectroscopy on borophene with a CO-functionalized tip. f CO-AFM image of $v_{1 / 5}$ phase borophene. $\mathbf{g}$ Simulated CO-AFM image of $v_{1 / 5}$ phase borophene with overlaid atomic structure. $\mathbf{h}$ CO-STM and $\mathbf{i}$ dynamic CO-STM image of $v_{1 / 5}$ phase borophene. The red arrows in $\mathbf{f}, \mathbf{h}$, and $\mathbf{i}$ denote the unit cells corresponding to the $\mathrm{HH}$ lattice. $V_{\mathrm{s}}=-10 \mathrm{~m} V$ in $\mathbf{a}, 20$ $\mathrm{mV}$ in b, $100 \mathrm{mV}$ in $\mathbf{d},-7 \mathrm{mV}$ in $\mathbf{h}$, and $15 \mathrm{mV}$ in i. Scale bars, $2 \mathrm{~nm}$ in a, $1 \mathrm{~nm}$ in $\mathbf{b}, 2 \AA$ in $\mathbf{d}, \mathbf{f}, \mathbf{h}, \mathbf{i}$

structure again agrees well with experimental observations, confirming our structural assignment as $v_{1 / 6}-30^{\circ}$. On the other hand, simulated CO-AFM images of a buckled triangular lattice deviate from the experimental image (Supplementary Fig. 3). Compared to the bare tip STM image, each bright protrusion splits into two protrusions with inequivalent brightness in the CO-STM image in Fig. 2h. A series of bare-tip STM, CO-AFM, and CO-STM images of a $60^{\circ} \mathrm{GB}$ in $v_{1 / 6}-30^{\circ}$ phase borophene is shown in Fig. 2i, j, l, respectively. Based on the apparent bonding positions resolved in Fig. 2j, we propose an atomic structure of the GB seamlessly connecting adjacent grains (Fig. 2k). This proposed structure yields a simulated CO-AFM image (Fig. 2k) that agrees well with experimental observation, further supporting our assignment of the $v_{1 / 6}-30^{\circ}$ phase.

As established previously, intermixing of $v_{1 / 5}$ and $v_{1 / 6}$ phase borophene takes place when the two phases have rotationally aligned $\mathrm{HH}$ rows ${ }^{7}$. Therefore, it is reasonable to expect that $v_{1 / 5}-30^{\circ}$ phase borophene (schematically shown in Fig. 3a) would intermix with the $v_{1 / 6}-30^{\circ}$ phase at appropriate growth conditions. Indeed, at a higher growth temperature $\left(\sim 550^{\circ} \mathrm{C}\right)$, an undulated structure appears as shown in the bare-tip STM image in Fig. 3b, which has previously been referred to as the striped phase $\mathrm{e}^{5}$ and suggested to be induced by an undulating Ag surface reconstruction under a $v_{1 / 6}-30^{\circ}$ sheet $^{28}$. However, based on the CO-STM image in Fig. $3 c$, we conclude that this phase adopts the $v_{1 / 5}$ borophene structure as evidenced by the staggered arrangement of protrusions (yellow circles) representing the $\mathrm{HH}$ lattice. Furthermore, instead of the brick-wall type Moiré pattern observed for the low-temperature $v_{1 / 5}$ phase, the apparent larger-scale undulation results from a $\sim 4 \%$ larger inter- $\mathrm{HH}$ spacing along the $\mathrm{HH}$ rows than the interatomic spacing of the underlying $\mathrm{Ag}$, which is reproduced qualitatively in Fig. $3 \mathrm{~d}$ by rotating $v_{1 / 5}$ phase borophene by $30^{\circ}$ on $\operatorname{Ag}(111)$. The coexistence of this structure with $v_{1 / 6}-30^{\circ}$ phase borophene with parallel $\mathrm{HH}$ rows and a seamless phase boundary is shown in the CO-STM image in Fig. 3e, confirming a rotation angle of $30^{\circ}$ and thus the identity of this phase as $v_{1 / 5}-30^{\circ}$. Since phase intermixing results from line defects in each phase adopting the structure of the other phase ${ }^{7}$, we identify various $v_{1 / 5}-30^{\circ}$ line defects in $v_{1 / 6}-30^{\circ}$ phase borophene with different widths as shown in the top row of Fig. $3 \mathrm{f}-\mathrm{i}$ (CO-STM images). The yellow circles denote the staggered $\mathrm{HH}$ pattern in the $v_{1 / 5}-30^{\circ}$ regions, while the corresponding structures are displayed in the bottom row. Additional CO-STM and CO-AFM images of intermixed $v_{1 / 6}-30^{\circ}$ and $v_{1 / 5}-30^{\circ}$ phase borophene are provided in Supplementary Fig. 4.

Rotationally incommensurate borophene phases. All of the borophene phases discussed thus far (i.e., $v_{1 / 6}, v_{1 / 5}, v_{1 / 6}-30^{\circ}$, and $v_{1 / 5}-30^{\circ}$ ) are reasonably characterized as being rotationally 
a

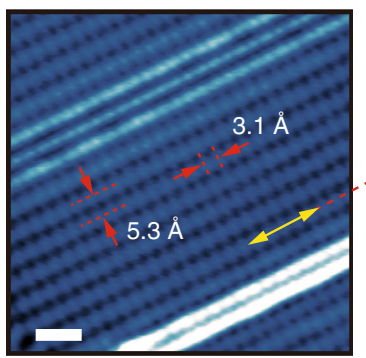

b

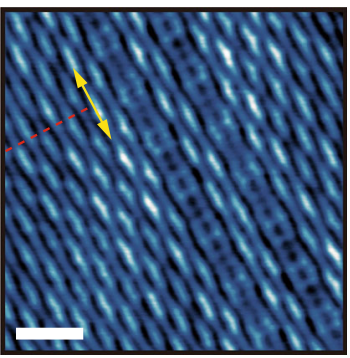

C

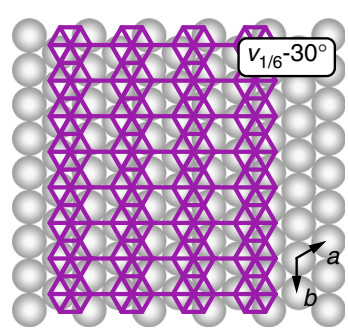

d

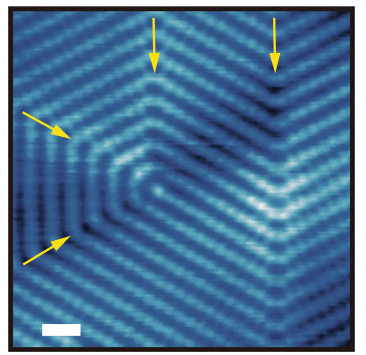

$\mathbf{e}$

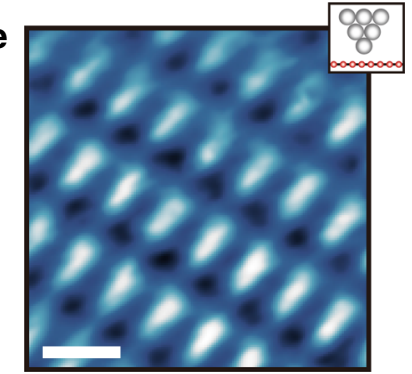

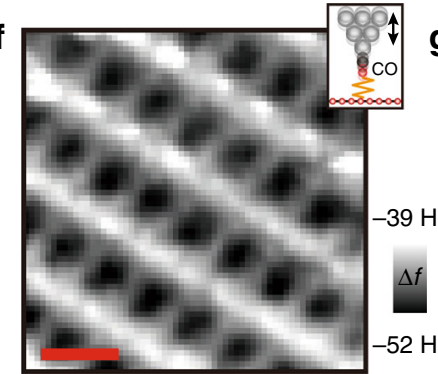
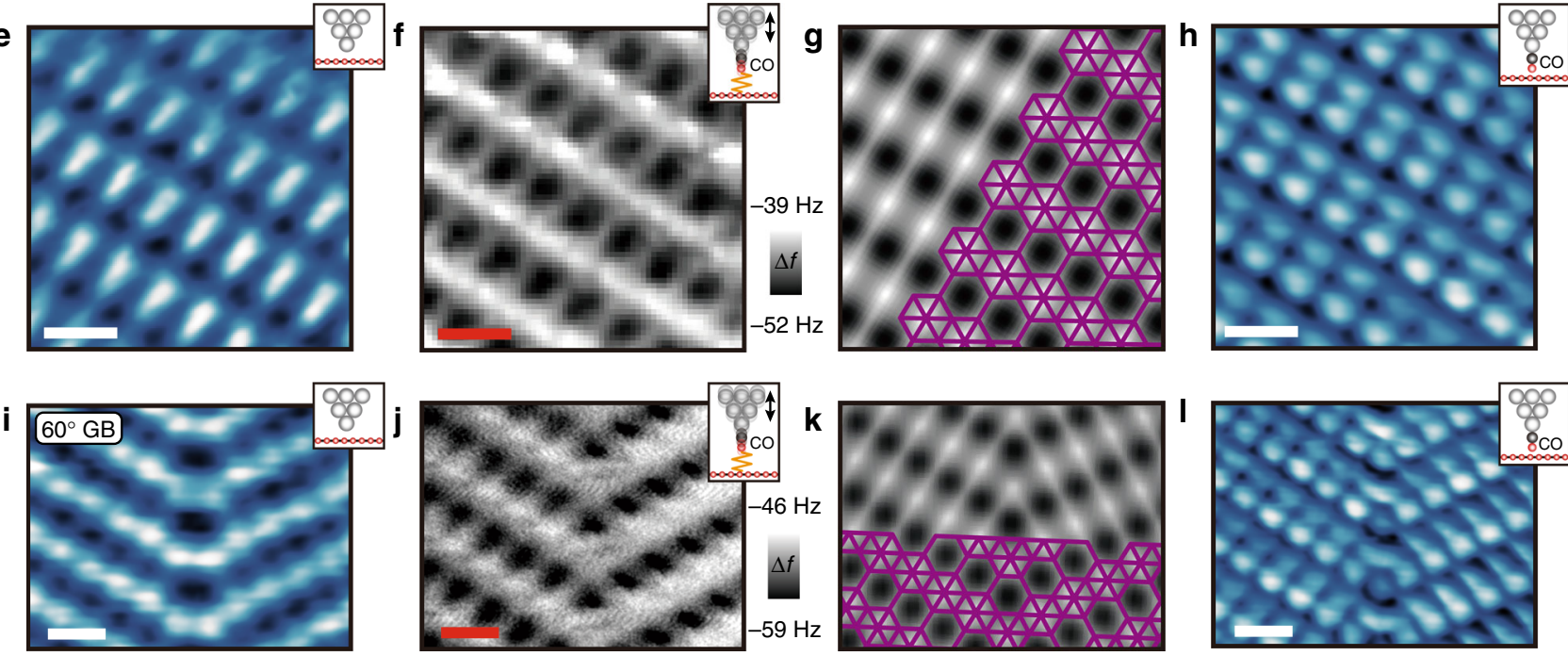

Fig. 2 Imaging $v_{1 / 6}-30^{\circ}$ phase borophene structures with bare and functionalized probes. Bare-tip STM images of a a $v_{1 / 6}-30^{\circ}$ phase borophene domain and $\mathbf{b}$ a neighboring $v_{1 / 5}$ phase borophene, where the $\mathrm{HH}$ rows in each phase are perpendicular (yellow arrows). $\mathbf{c}$ Schematic of $v_{1 / 6}-30^{\circ}$ phase borophene. d Bare-tip STM image of polycrystalline $v_{1 / 6}-30^{\circ}$ phase borophene with multiple $60^{\circ} \mathrm{GBs}$ (yellow arrows). e Bare-tip, $\mathbf{f} \mathrm{CO}-\mathrm{AFM}, \mathbf{g}$ simulated CO-AFM, and $\mathbf{h}$ CO-STM images of $v_{1 / 6}-30^{\circ}$ phase borophene. The atomic structure is overlaid in $\mathbf{g}$. i Bare-tip, $\mathbf{j}$ CO-AFM, $\mathbf{k}$ simulated CO-AFM, and $\mathbf{I}$ CO-STM images of a $60^{\circ} \mathrm{GB}$ within $V_{1 / 6}-30^{\circ}$ phase borophene. The atomic structure is overlaid in $\mathbf{k} . V_{\mathrm{s}}=-3 \mathrm{mV}$ in $\mathbf{a}, 4 \mathrm{mV}$ in $\mathbf{b},-34 \mathrm{mV}$ in $\mathbf{d}, 9 \mathrm{mV}$ in $\mathbf{e}, 4 \mathrm{mV}$ in $\mathbf{h},-34 \mathrm{mV}$ in $\mathbf{i}$, and $4 \mathrm{mV}$ in $\mathbf{I}$. Scale bars, $1 \mathrm{~nm}$ in $\mathbf{a}, 2 \mathrm{~nm}$ in $\mathbf{b}, 1 \mathrm{~nm}$ in $\mathbf{d}, 5 \AA$ in $\mathbf{e}, \mathbf{f}, \mathbf{h}-\mathbf{j}, \mathbf{I}$
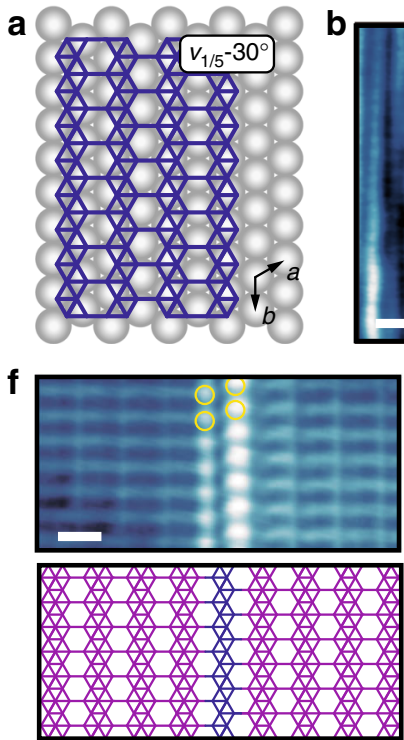

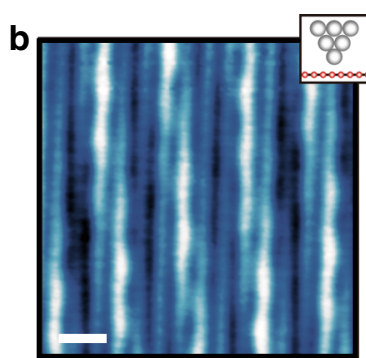

9
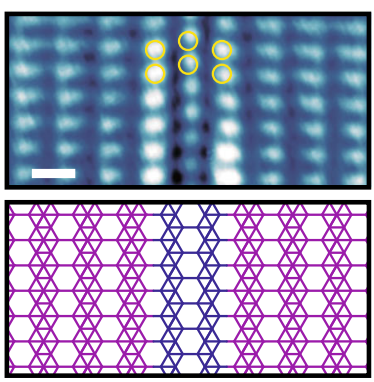

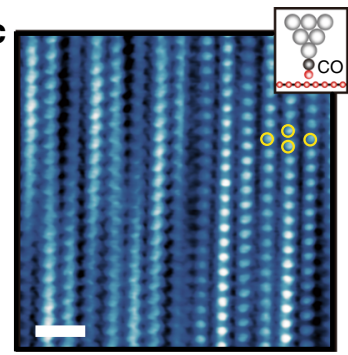

$\mathbf{h}$
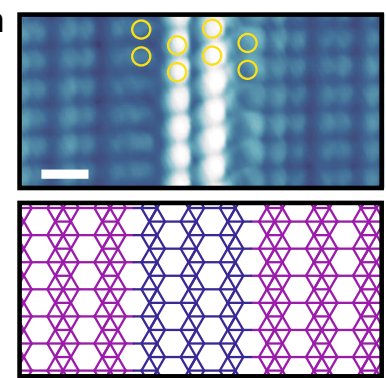
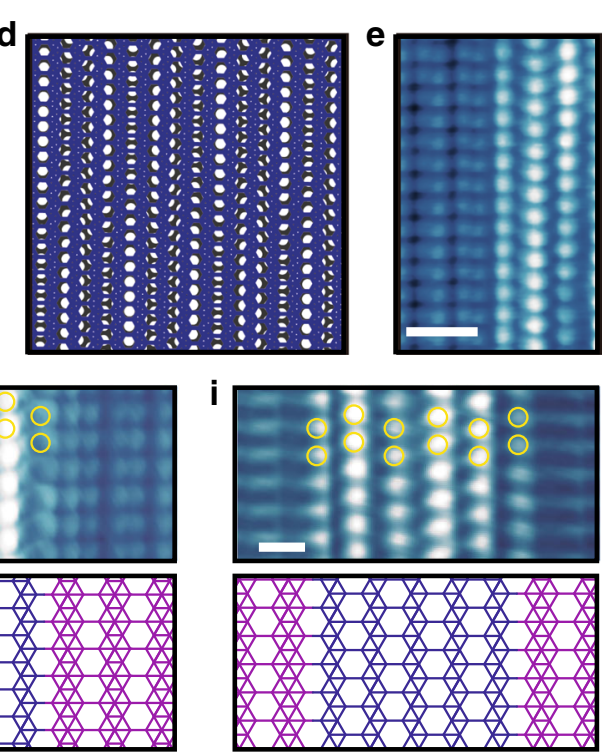

Fig. 3 Resolving $v_{1 / 5}-30^{\circ}$ phase borophene and its intermixing with the $v_{1 / 6}-30^{\circ}$ phase. a Schematic of $v_{1 / 5}-30^{\circ}$ phase borophene. b Bare-tip, and c CO-STM images of undulating $v_{1 / 5}-30^{\circ}$ phase borophene. The yellow circles in $\mathbf{c}$ indicate the staggered $\mathrm{HH}$ pattern. $\mathbf{d}$ Schematic of the undulations resulting from a lattice mismatch between borophene and $\mathrm{Ag}(111)$. e CO-STM image of the phase boundary between $v_{1 / 6}-30^{\circ}$ (left) and $v_{1 / 5}-30^{\circ}$ phase borophene. $\mathbf{f}-\mathbf{i}$ CO-STM images of $v_{1 / 5}-30^{\circ}$ line defects in $v_{1 / 6}-30^{\circ}$ phase borophene with different widths. The yellow circles denote the staggered arrangement of the $\mathrm{HH}$ patterns, with the corresponding structure schematics provided in the bottom row. $V_{\mathrm{s}}=-3 \mathrm{mV}$ in $\mathbf{b}, \mathbf{c},-7 \mathrm{mV}$ in $\mathbf{e}, \mathbf{f},-1 \mathrm{mV}$ in $\mathbf{g}$, $4 \mathrm{mV}$ in $\mathbf{h}$, and $-7 \mathrm{mV}$ in i. Scale bars, $1 \mathrm{~nm}$ in $\mathbf{b}, \mathbf{c}, \mathbf{e}, 5 \AA$ in $\mathbf{f}-\mathbf{i}$ 

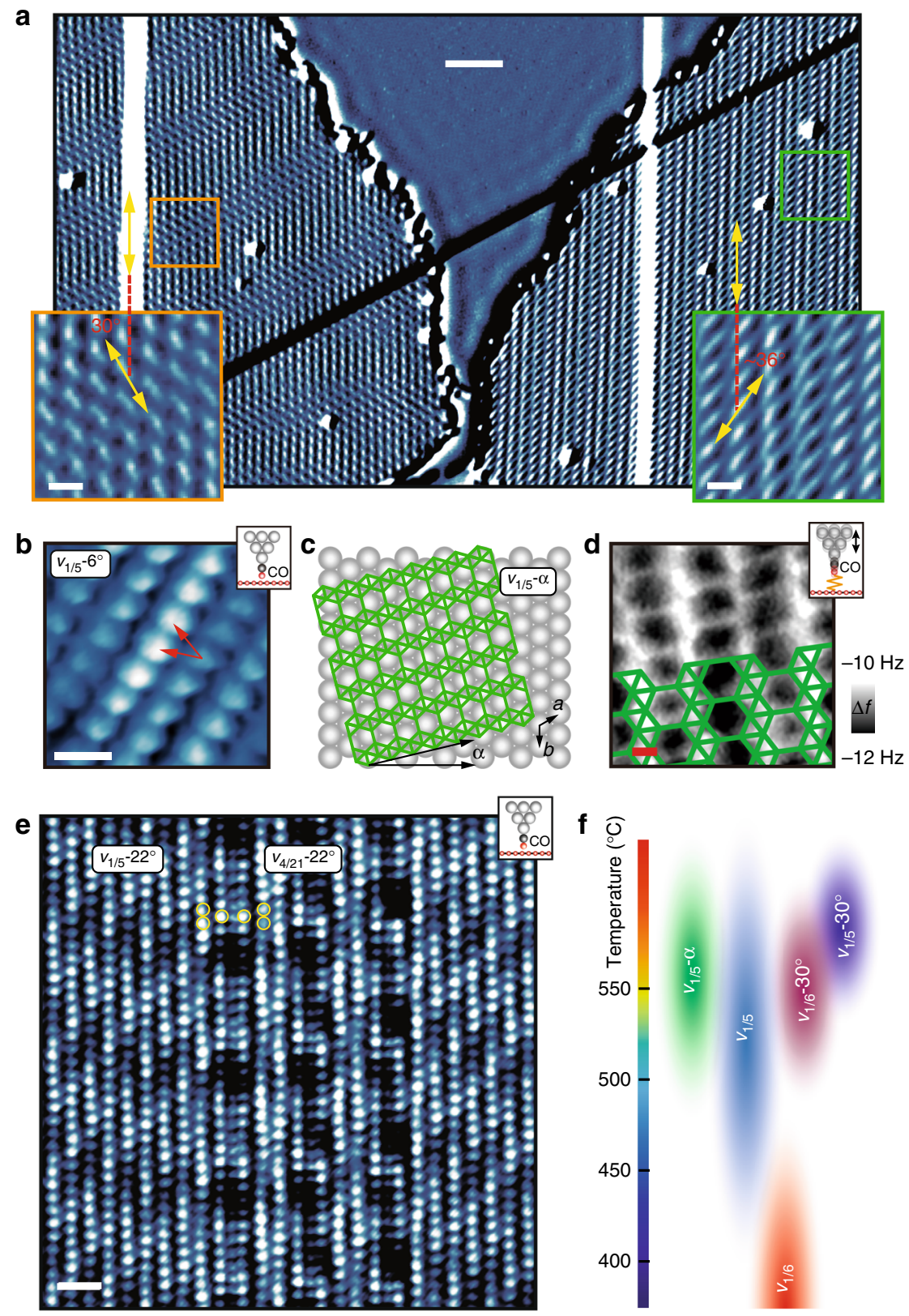

Fig. 4 Imaging rotationally incommensurate phases of borophene with functionalized probes. a Large-scale derivative image of the bare-tip STM topography for $v_{1 / 5}$ (left) and $v_{1 / 5}-6^{\circ}$ (right) phase borophene domains. The insets are the zoomed-in images in the orange and green squares. The relative orientations of the $\mathrm{HH}$ rows in each phase with respect to the $\mathrm{Ag}$ atomic chains (vertical direction) are labeled. $\mathbf{b} \mathrm{CO}-\mathrm{STM}$ image of $v_{1 / 5}-6^{\circ}$ phase borophene, where the red arrows indicate the staggered $\mathrm{HH}$ lattice pattern. c Schematic of rotationally incommensurate borophene phases, where $\alpha$ denotes the angle by which the sheet is rotated from $v_{1 / 5}$ phase borophene. d CO-AFM image of $v_{1 / 5}-6^{\circ}$ phase borophene with overlaid atomic structure. e CO-STM image of $v_{1 / 5}-22^{\circ}$ phase borophene with self-assembled $v_{1 / 6}-22^{\circ}$ line defects. The yellow circles denote the aligned and staggered $\mathrm{HH}$ patterns. f Phase diagram of borophene with respect to growth temperature. Individual ovals represent each phase. $V_{\mathrm{s}}=190 \mathrm{mV}$ in $\mathbf{a},-2 \mathrm{mV}$ in $\mathbf{b}$, and 22 $\mathrm{mV}$ in e. Scale bars, $5 \mathrm{~nm}$ in $\mathbf{a}, 1 \mathrm{~nm}$ in the insets in $\mathbf{a}, 5 \AA$ in $\mathbf{b}, 2 \AA$ in $\mathbf{d}, 1 \mathrm{~nm}$ in $\mathbf{e}$

commensurate with the underlying growth substrate since the $\mathrm{HH}$ rows are aligned along high symmetry directions of the $\operatorname{Ag}(111)$ surface. In contrast, at similar conditions where phase intermixing is observed for $v_{1 / 6}-30^{\circ}$ and $v_{1 / 5}-30^{\circ}$ borophene, another new structure is observed as shown in the derivative image of the bare-tip STM topography in Fig. 4a. As seen in the zoomed-in inset images, the borophene domain on the left is $v_{1 / 5}$ phase borophene with a characteristic brick-wall type Moiré pattern (orange square), whereas the domain on the right shows a distorted brick-wall type Moiré pattern (green square). The CO-STM image of this new domain structure is provided in Fig. $4 \mathrm{~b}$ and reveals a $\mathrm{HH}$ lattice with staggered protrusions (red arrows) that are indicative of a $v_{1 / 5}$ borophene sheet. However, due to the $\sim 36^{\circ}$ angle (instead of $30^{\circ}$ angle for $v_{1 / 5}$ ) between the directions of the $\mathrm{HH}$ rows and the Ag atomic chains (vertical direction, Fig. 4a), this borophene sheet is denoted as $v_{1 / 5}-6^{\circ}$. Rotationally incommensurate $v_{1 / 5}$ borophene sheets are generally depicted in Fig. $4 c$ as $v_{1 / 5^{-}} \alpha$, where $\alpha$ is the rotation angle by which the sheet deviates from the $v_{1 / 5}$ phase. In Fig. 4d, a CO-AFM image of the $v_{1 / 5}-6^{\circ}$ phase with an overlaid atomic structure confirms the assignment as a $v_{1 / 5}$ sheet. 
In addition to $\alpha=6^{\circ}$, other rotationally incommensurate phases such as $v_{1 / 5}-9^{\circ}$ and $v_{1 / 5}-22^{\circ}$ phase borophene have been observed (Supplementary Fig. 5). As an example, the CO-STM image in Fig. $4 \mathrm{e}$ resolves the $\mathrm{HH}$ lattice of $v_{1 / 5}-22^{\circ}$ phase borophene with periodically self-assembled $v_{1 / 6}-22^{\circ}$ line defects in the middle, as evidenced by the staggered and aligned $\mathrm{HH}$ patterns indicated by the yellow circles, respectively. Equivalently, the self-assembled periodic line defects can be categorized as $v_{4 / 21}-22^{\circ}$ phase borophene (another example is shown in Supplementary Fig. 5) in a manner analogous to previously reported periodic intermixed phases for non-rotated borophene growth (i.e., $\left.\alpha=0^{\circ}\right)^{7}$. Combining all of the observations discussed above and established previously, a phase diagram for borophene growth with respect to temperature is summarized in Fig. 4f, where each phase is represented by an oval. The overall trend is that borophene growth transitions from rotationally commensurate phases to rotationally incommensurate phases at high temperatures. Significantly, the existence of rotationally incommensurate phases provides corroborating evidence that borophene layers are chemically discrete from the underlying Ag surface ${ }^{8}$. It should be further noted that the range of observed borophene phases show similar electronic properties (Supplementary Fig. 6) with no measurable differences in lattice constants.

In summary, we have utilized CO-functionalized scanning probe microscopy to geometrically image and determine the atomic lattice structures of various borophene polymorphs. In addition to CO-AFM, we establish CO-STM as an alternative and comparatively more accessible technique for unambiguously determining borophene atomic structures. Using these methods, we assigned structure models for several phases of borophene in addition to resolving features consistent with boron-boron covalent bonds. In all cases, the borophene phases are found to consist of $v_{1 / 5}$ and/or $v_{1 / 6}$ domains, although the orientation of these domains with respect to the underlying $\operatorname{Ag}(111)$ substrate transitions from rotationally commensurate to rotationally incommensurate at the highest growth temperatures. The resulting phase diagram explains all observations of borophene to date with the newest rotationally incommensurate phases providing strong evidence that borophene is a chemically discrete two-dimensional material as opposed to a surface reconstruction or alloy with the underlying $\operatorname{Ag}(111)$ growth substrate. This conclusion is further supported by the recent synthesis of borophene on $\mathrm{Cu}(111)^{30}$. The ability of CO-AFM and CO-STM to resolve the complicated phase diagram for borophene polymorphs suggests that CO-functionalized probes can be similarly employed for the unambiguous determination of atomic structures for other emerging synthetic $2 \mathrm{D}$ materials and their heterostructures ${ }^{31}$.

\section{Methods}

Borophene growth. Borophene growth is described in detail in an earlier report ${ }^{7}$. Briefly, a solid boron rod (ESPI metals, $99.9999 \%$ purity) is evaporated onto Ag (111) films ( $\sim 60 \mathrm{~nm}$ thick) on mica (Princeton Scientific Corp.) in an ultrahigh vacuum preparation chamber $\left(\sim 1 \times 10^{-10} \mathrm{mbar}\right)$ with an electron-beam evaporator (FOCUS) for a duration of $\sim 30 \mathrm{~min}$ to achieve submonolayer coverage. The clean $\mathrm{Ag}(111)$ surface for borophene growth is prepared by repeated $30 \mathrm{~min} \mathrm{Ar}$ ion sputtering at $1 \times 10^{-5} \mathrm{mbar}$ followed by $30 \mathrm{~min}$ annealing at $550{ }^{\circ} \mathrm{C}$.

Scanning probe microscopy and tip-functionalization. Low temperature STM/ STS and non-contact AFM characterization is performed on a Scienta Omicron LT STM $\left(\sim 2 \times 10^{-11}\right.$ mbar) at $\sim 4 \mathrm{~K}$ interfaced with Nanonis (SPECS) control electronics using qPlus AFM sensors (mounted W tips from Scienta Omicron). Tip functionalization is achieved by leaking CO molecules into the STM chamber $\left(1 \times 10^{-7}\right.$ mbar for $\left.40 \mathrm{~s}\right)$ and picking up individual CO molecules by ramping the sample bias down to $-2 \mathrm{mV}$ and the tunneling current up to $1 \mathrm{nA}$. For AFM measurements, an oscillation amplitude of $1-2 \AA$ is used. AFM image simulation is based on the method developed by Hapala and coworkers ${ }^{24,25}$. A lock-in amplifier (SRS model SR850) is used for STS measurements with $2 \mathrm{mV}_{\mathrm{RMS}}$ amplitude and $\sim 0.8 \mathrm{kHz}$ modulation frequency. Gwyddion software is used for image processing.

\section{Data availability}

All data needed to evaluate the conclusions in the paper are present in the paper and/or the Supplementary Information. Additional data related to this paper may be requested from the authors.

Received: 4 January 2019 Accepted: 22 March 2019

Published online: 09 April 2019

\section{References}

1. Mannix, A. J., Zhang, Z., Guisinger, N. P., Yakobson, B. I. \& Hersam, M. C. Borophene as a prototype for synthetic $2 \mathrm{D}$ materials development. Nat. Nanotechnol. 13, 444-450 (2018).

2. Zhang, Z., Penev, E. S. \& Yakobson, B. I. Two-dimensional materials: polyphony in B flat. Nat. Chem. 8, 525-527 (2016).

3. Penev, E. S., Bhowmick, S., Sadrzadeh, A. \& Yakobson, B. I. Polymorphism of two-dimensional boron. Nano Lett. 12, 2441-2445 (2012).

4. Feng, B. et al. Experimental realization of two-dimensional boron sheets. Nat. Chem. 8, 563-568 (2016).

5. Mannix, A. J. et al. Synthesis of borophenes: anisotropic, two-dimensional boron polymorphs. Science 350, 1513-1516 (2015).

6. Liu, X. et al. Self-assembly of electronically abrupt borophene/organic lateral heterostructures. Sci. Adv. 3, e1602356 (2017).

7. Liu, X., Zhang, Z., Wang, L., Yakobson, B. I. \& Hersam, M. C. Intermixing and periodic self-assembly of borophene line defects. Nat. Mater. 17, 783-788 (2018).

8. Campbell, G. P. et al. Resolving the chemically discrete structure of synthetic borophene polymorphs. Nano Lett. 18, 2816-2821 (2018).

9. Zhang, Z., Yang, Y., Penev, E. S. \& Yakobson, B. I. Elasticity, flexibility, and ideal strength of borophenes. Adv. Funct. Mater. 27, 1605059 (2017).

10. Feng, B. et al. Dirac fermions in borophene. Phys. Rev. Lett. 118, 096401 (2017).

11. Luo, Z., Fan, X. \& An, Y. First-principles study on the stability and STM image of borophene. Nanoscale Res. Lett. 12, 514 (2017).

12. Repp, J., Meyer, G., Stojković, S. M., Gourdon, A. \& Joachim, C. Molecules on insulating films: scanning-tunneling microscopy imaging of individual molecular orbitals. Phys. Rev. Lett. 94, 026803 (2005).

13. Gross, L., Mohn, F., Moll, N., Liljeroth, P. \& Meyer, G. The chemical structure of a molecule resolved by atomic force microscopy. Science 325, 1110-1114 (2009).

14. Temirov, R., Soubatch, S., Neucheva, O., Lassise, A. C. \& Tautz, F. S. A novel method achieving ultra-high geometrical resolution in scanning tunnelling microscopy. New J. Phys. 10, 053012 (2008).

15. Martinez, J. I., Abad, E., González, C., Flores, F. \& Ortega, J. Improvement of scanning tunneling microscopy resolution with H-sensitized tips. Phys. Rev. Lett. 108, 246102 (2012).

16. Hapala, P. et al. Mapping the electrostatic force field of single molecules from high-resolution scanning probe images. Nat. Commun. 7, 11560 (2016).

17. Moenig, H. et al. Submolecular imaging by noncontact atomic force microscopy with an oxygen atom rigidly connected to a metallic probe. ACS Nano 10, 1201-1209 (2016).

18. Mönig, H. et al. Quantitative assessment of intermolecular interactions by atomic force microscopy imaging using copper oxide tips. Nat. Nanotechnol. 13, 371-375 (2018).

19. Hämäläinen, S. K. et al. Intermolecular contrast in atomic force microscopy images without intermolecular bonds. Phys. Rev. Lett. 113, 186102 (2014).

20. Zhang, J. et al. Real-space identification of intermolecular bonding with atomic force microscopy. Science 342, 611-614 (2013).

21. Han, Z. et al. Imaging the halogen bond in self-assembled halogenbenzenes on silver. Science 358, 206-210 (2017).

22. de la Torre, B. et al. Submolecular resolution by variation of the inelastic electron tunneling spectroscopy amplitude and its relation to the AFM/STM signal. Phys. Rev. Lett. 119, 166001 (2017)

23. Han, Z. et al. Probing intermolecular coupled vibrations between two molecules. Phys. Rev. Lett. 118, 036801 (2017).

24. Hapala, P. et al. Mechanism of high-resolution STM/AFM imaging with functionalized tips. Phys. Rev. B 90, 085421 (2014).

25. Hapala, P., Temirov, R., Tautz, F. S. \& Jelínek, P. Origin of high-resolution IETS-STM images of organic molecules with functionalized tips. Phys. Rev. Lett. 113, 226101 (2014).

26. Liu, M. et al. Graphene-like nanoribbons periodically embedded with fourand eight-membered rings. Nat. Commun. 8, 14924 (2017).

27. Herz, M., Giessibl, F. J. \& Mannhar, J. Probing the shape of atoms in real space. Phys. Rev. B 68, 045301 (2003).

28. Zhang, Z. et al. Substrate-induced nanoscale undulations of borophene on silver. Nano Lett. 16, 6622-6627 (2016). 
29. Zhong, Q. et al. Metastable phases of 2D boron sheets on $\mathrm{Ag}(111)$. J. Phys. Condens. Matter. 29, 095002 (2017).

30. $\mathrm{Wu}$, R. et al. Large-area single-crystal sheets of borophene on $\mathrm{Cu}(111)$ surfaces. Nat. Nanotechnol. 14, 44-49 (2019).

31. Mannix, A. J., Kiraly, B., Hersam, M. C. \& Guisinger, N. P. Synthesis and chemistry of elemental 2D materials. Nat. Rev. Chem. 1, 0014 (2017).

\section{Acknowledgements}

The experimental work was supported by the Office of Naval Research (ONR N0001417-1-2993) and the National Science Foundation Materials Research Science and Engineering Center (NSF DMR-1720139). The computational modeling was supported by the US DOE Office of Science (DOE DE-SC0012547). X.L. further acknowledges support from a Ryan Fellowship that is administered through the Northwestern University International Institute for Nanotechnology. The authors kindly thank Ruslan Temirov for valuable discussions.

\section{Author contributions}

X.L. and M.C.H. conceived the experiments. X.L. performed sample preparation, STM/ STS/AFM characterization, and image simulations. L.W. and B.I.Y. provided theoretical insights and structure models. S.L and M.S.R. provided experimental assistance. All authors contributed to data interpretation and manuscript writing.

\section{Additional information}

Supplementary Information accompanies this paper at https://doi.org/10.1038/s41467019-09686-w.
Competing interests: The authors declare no competing interests.

Reprints and permission information is available online at http://npg.nature.com/ reprintsandpermissions/

Journal peer review information: Nature Communications would like to thank Han Woong Yeom, and the anonymous reviewers for their contributions to the peer review of this work. Peer review reports are available.

Publisher's note: Springer Nature remains neutral with regard to jurisdictional claims in published maps and institutional affiliations.

(c) (i) Open Access This article is licensed under a Creative Commons Attribution 4.0 International License, which permits use, sharing, adaptation, distribution and reproduction in any medium or format, as long as you give appropriate credit to the original author(s) and the source, provide a link to the Creative Commons license, and indicate if changes were made. The images or other third party material in this article are included in the article's Creative Commons license, unless indicated otherwise in a credit line to the material. If material is not included in the article's Creative Commons license and your intended use is not permitted by statutory regulation or exceeds the permitted use, you will need to obtain permission directly from the copyright holder. To view a copy of this license, visit http://creativecommons.org/ licenses/by/4.0/.

(C) The Author(s) 2019 